recorrido por la figura del arquitecto que proyectó el antiguo Mercado de Mayoristas, Luis Gutiérrez Soto, el estado en que se encontraba el edificio que fue mercado entre 1942 y 1980, las patologías que presentaba, su tratamiento técnico, y el análisis del proyecto de rehabilitación del edificio del que son responsables.

En la siguiente sesión Francisco Luque, Doctor en Historia del Arte, en su conferencia "El espacio turístico: intervención", desarrolló algunos aspectos polémicos sobre las intervenciones en núcleos patrimoniales y su conversión en focos de atracción turistica.

Rosa Domínguez, profesora del "Master Internacional de Desarrollo Local" de la Fundación Infodal-Fundación Carolina, presentó su ponencia "Centros culturales como dinamizadores de ciudad". En ella elaboró un hilo argumental basado en la demostración, a través de ejemplos prácticos, de que no siempre los centros culturales son útiles para dinamizar social, cultural y económicamente la ciudad, y que esto sólo se logra cuando se articulan pautas o claves de "interpretación" del Patrimonio, que consiguen crear el vínculo y/o implicar a la población autóctona y foránea.

El arquitecto y conservador del Museo Nacional de Antropología, Juan Carlos Rico, en dos sesiones de trabajo denominadas "Investigación y equipamiento cultural. Propuestas de vanguardia" e "Info-arquitecturas: propuestas experimentales para público y cultura", planteó la necesidad de afrontar los retos que presentan los museos y centros de exposiciones del presente siglo XXI, utilizando como instrumentos básicos la investigación y la experimentación. Desde la aplicación de esta metodología de trabajo, presentó buena parte de los proyectos, fruto de las últimas investigaciones sobre el museo del futuro realizadas por su equipo de trabajo integrado por arquitectos y estudiantes de arquitectura, que han sido recogidos también en su libro de reciente publicación: La difícil supervivencia del museo.

Suscitaron notable interés las dos visitas realizadas al Museo Picasso que fueron guiadas aplicando nuevas formas de percepción y análisis de la obra de arte -"Visual Thinking Strategies: aprendiendo a pensar y comunicarse a través del arte"-, metodología adquirida por el personal del Departamento de Didáctica del Museo en una consultora norteamericana dedicada a la formación de formadores, que desarrolla, en el ámbito de la cultura, programas como el denominado "Visual Understanding in Education".

Rosa Domínguez González

Coordinadora cientifica

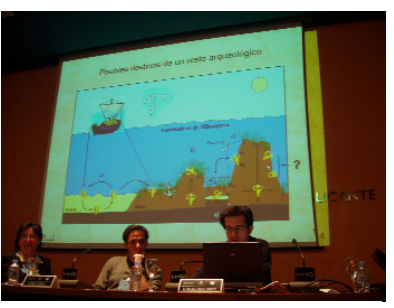

Reconstrucción ideal de los procesos de deposición de materiales arqueológicos en la pradera de Posidonia oceanica por M.A. Mateo Foto J.A. Moya- Taller Imagen-UA)

\title{
Se refuerza la cooperación en torno a los antiguos puertos y fondeaderos del Mediterráneo Occidental
}

Dentro de la fase de intercambio de experiencias del proyecto europeo ANSER, Antiguas Rutas Maritimas del Mediterráneo (véase $\mathrm{PH} 46, \mathrm{p} .11$ ), ha comenzado el ciclo de seminarios sobre El patrimonio arqueológico submarino y los puertos antiguos. El primero de ellos, titulado Evolución paleoambiental de los puertos y fondeaderos antiguos en el Mediterráneo Occidental, tuvo lugar en el Museo Arqueológico Provincial de Alicante (MARQ) los días 14 y 15 de noviembre de 2003. En él participaron destacados investigadores procedentes de Italia, Francia, Portugal, Argelia, Marruecos, Malta y España.

Las dos jornadas de trabajo se estructuraron en bloques de varias ponencias, seguidos de un breve turno de preguntas y respuestas. Se comenzó con tres ponencias relativas a la antropización y sedimentación marina en Marsella (A. Hesnard et alii), el paleoambiente del puerto de Alejandría (J-P. Goiran) y el paísaje litoral mediterráneo del Magreb (M. Betrouni leída por S. Ferdi). Tras la pausa se trató de paleogeografía y actividades portuarias en Portugal (M.L. P. Blot), paleogeografía costera en Etruria (M. Pasquinucci) y el marco geomorfológico de la costa toscana (E. Pranzini y S. Silenzi).
Por la tarde se inició la sesión con un primer bloque de ponencias sobre la transformación de la costa en las Islas Pontinas (A. Zarattini) y el nivel del mar a través de las estructuras arqueológicas en la costa tirrénica ( $M$. Anzidei, A. Benini et alii). A continuación se habló de la arqueología litoral maltesa (T. Gambin) y el estudio de la evolución en la costa ligur (T. Mannoni).

La segunda jornada, dedicada al área mediterránea de la Península Ibérica, comenzó con dos ponencias sobre la paleotopografía costera antigua del litoral gaditano (C. Alonso y F. J. Gracia) y las posibilidades de la Posidonia oceanica como archivo orgánico milenario (M. A. Mateo y su equipo), centrándose las tres siguientes en el análisis de esta problemática dentro del ámbito de la Comunidad Valenciana: la paleoribera protohistórica de la desembocadura del Segura (P. Rouillard, P. Barrier y C. Montenat); la paleografía y geoarqueología en la zona del Turia y Júcar (P. Carmona y J.M. Ruiz) y los viveros de peces y las salinas en el litoral (V. Rosselló).

Este primer seminario finalizó con una mesa redonda, compuesta por $\mathrm{R}$. Turchetti, coordina- 
$018-019$

\section{Noticias y comentarios}

\section{PH47 - Febrero 2004}

dora del ciclo de seminarios, A. Hesnard de la región francesa PACA, A. Akerraz de Marruecos, T. Mannoni de la Liguria y A. Fernández de la Comunidad Valenciana, que comentaron las conclusiones de las ponencias. En definitiva, se ha conseguido tener una visión más completa y actualizada de la evolución paleoambiental de la línea de costa, así como de los puertos y fondeaderos antiguos del Mediterráneo Occidental.

Como actividad complementaria se realizó una visita guiada al museo, recorriendo tanto las salas de exposición como el resto de sus instalaciones (biblioteca, almacenes -ingresos y salidas, anforario, compactos, almacén visitable -, área de restauración...).

El gran rendimiento del seminario no se mide sólo por el alto nivel científico de las ponencias, sino también por sus resultados indirectos. Las conversaciones paralelas han dado lugar a la creación espontánea de nuevos e interesantes grupos de trabajo sobre la aplicación del estudio de la Posidonia oceanica en la arqueología maritima; los paralelos entre los viveros de peces en la costa tirrénica y alicantina en la antigüedad; etc. Todo ello redunda en la intensificación de las relaciones entre los miembros de las distintas regiones integrantes y en la creación de redes estables entre sus investigadores para el estudio en el marco del Mediterráneo Occidental, principal objetivo de la fase B del proyecto ANSER.

A lo largo del año 2004 se celebrarán los siguientes seminarios: La estructura de los puertos y fondeaderos antiguos (Roma, 12-13 marzo 2004); Las actividades humanas de los puertos y fondeaderos antiguos (Marsella, 1415 mayo 2004); La historia de las rutas maritimas en el Mediterráneo (Génova, 18-19 junio 2004); y por último La puesta en valor del patrimonio arqueológico ligada a la presencia de los puertos y fondeaderos antiguos (Pisa, 17-18 septiembre 2004).

Más información:

El programa completo y los resúmenes de las ponencias ya se pueden consultar en la página web del proyecto www.projetanser.net

Las actas se publicarán durante el año 2004.

Mercedes Navarro Tito

Rocío Castillo Belinchón

Museo Arqueológico Provincial de Alicante

\section{Más de 6.000 visitantes celebraron las Jornadas Europeas de Patrimonio en Andalucía}

Como se informó en PH 45 (p.7), la Consejería andaluza de Cultura celebró el pasado 25 de octubre las Jornadas Europeas de Patrimonio, con una simbólica jornada de puertas abiertas de los edificios históricos rehabilitados para uso público en Andalucía, a la que se sumaron 6.494 visitantes.

En las ocho provincias andaluzas, la cifra de inmuebles visitables con ocasión de las Jornadas ascendió a 106, distribuidos según diversas tipologías originales (casas palacio, casas burguesas, edificaciones religiosas, defensivas, industriales...). En cuanto a su función actual, la mayoría apunta hacia el ámbito cultural -por razones de adaptación- o institucional -por el alto valor representativo que poseen: 25 inmuebles presentan carácter museístico, 21 son centros o casas de cultura, 19 dependencias municipales, 16 sedes de la Junta de Andalucía, 10 archivos o bibliotecas, 9 equipamientos o edificios docentes, 4 equipamientos escénicos, y 2 de hostelería.

Paralelamente a las Jornadas, la Consejería de Cultura ha publicado el título Edificios históricos rehabilitados de uso público en Andalucía, en el que se presenta una selección de los inmuebles visitados que permite cono- cer sus posibilidades de utilización social, y una concienciación y sensibilización de la ciudadanía sobre su necesidad de preservación, mediante un uso adecuado que garantice su futuro.

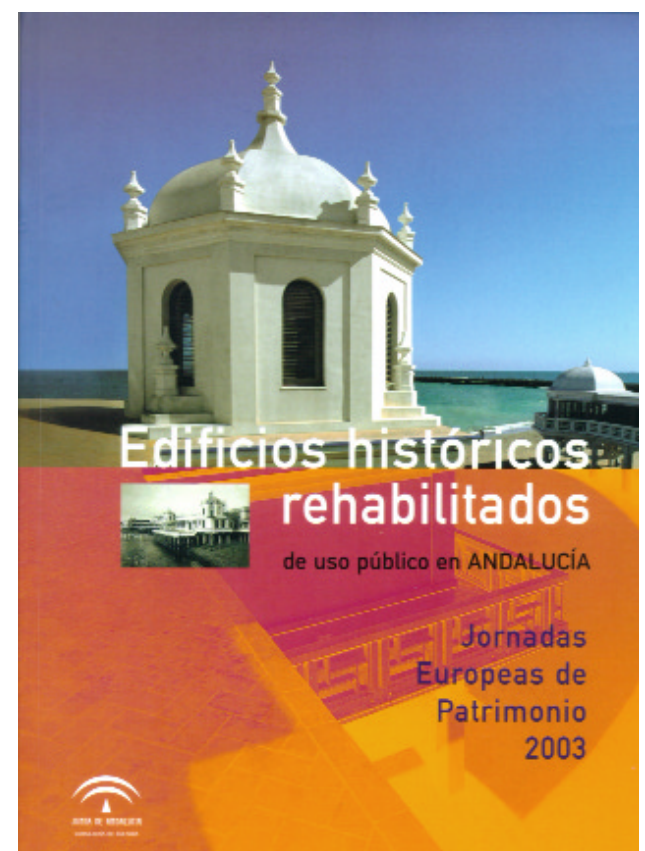

